\title{
Properties of Projection Lines in the Space of the Orientation Distribution Function
}

\author{
A. MORAWIEC and J. POSPIECH \\ Institute for Metal Research, Polish Academy of Sciences, Cracow
}

(Received February 1, 1988; in final form December 10, 1988)

The relationship between the orientation distribution function (ODF) and the pole figure is based on the geometry of projection lines in the orientation space.

The paper presents an analytical description of the projection lines and their transformations by symmetry operations. Using simple algebraical rules some properties of the projection lines as well as some properties of the associated projection lines (coupled due to the centrosymmetry of the pole figure) have been derived.

KEY WORDS: Euler space, symmetries, projection lines, pole figures.

\section{INTRODUCTION}

The basis for the reproduction of the ODF from pole figures is an integral equation with the integral along integration line running through the orientation space. When the method of series expansions (Bunge, 1969) was in common use, there was no need to analyse the course of these lines. It is different in case of the recently preferred direct methods in which the integral equation is approximated by system of equations connecting discrete values of pole figures with sums of discrete values of the ODF ascribed (with appropriate weights) to tubes in the orientation space.

The integration lines which are the idealization of tubes are 
(according to the interpretation of the integral equation) lines of projection of the ODF on the sphere of the pole figure. The projection lines appear as interesting, in particular their geometry in the space of Euler angles in which the ODF is described. The authors' aim is to present the basic properties of the projections lines, mainly those which are essential in problems of approximation of the ODF.

\section{EQUATION OF THE PROJECTION LINE}

The orientation distribution function is determined on the group of orthogonal transformations $(\mathrm{O}(3))$ (Esling et al., 1980). (We shall identify the rotation group with corresponding matrices of orthogonal group.) For the sake of simplicity we shall assume here that the inversion centre is an element of crystal symmetry (and hence the inversion centre belongs to sample symmetry group). The domain of the function can be then restricted to the space of proper rotations $(\mathrm{SO}(3))$.

A basis for the analysis of the problem of ODF calculation is an equation relating this function with the projection $P_{h}^{0}$

$$
P_{h}^{0}(y)=\frac{1}{2 \pi} \int_{[h, y]} f(g) d \gamma .
$$

The essence of this equation is as follows: the value of the function $P_{h}^{0}$ at the point $y$ is equal to the relative volume of the crystallites oriented in such a way that the vector $h$ established in the crystallite coordinate system coincides with the vector $y$ chosen in the sample coordinate system. In other words, it is equal to the sum of the densities of all these orientations which have a certain distinguished (in the crystallite coordinate system) vector $h$ as common and equal to $y$. Since it is assumed that the distribution of crystallites is continuous, the above mentioned sum is represented by an integral.

The relation between the coordinates of the vector $y$ and that of the vector $h$ in the sample and in the crystallite cartesian systems, respectively

$$
h_{i}=\sum_{j=1}^{3} g_{i j} y_{j}
$$


is virtually, with established coordinates of both these vectors, an equation of the projection line. It will be denoted by $[h, y]$. (This relation is usually written briefly as $h=g y$ and it will be used below.)

The orientation of a crystallite in a polycrystalline sample is determined by three Euler angles $\varphi_{1}, \phi, \varphi_{2}$ describing the rotation $g=\tilde{g}\left(\varphi_{1}, \phi, \varphi_{2}\right)$ of a sample coordinate system leading to its coincidence with the coordinate system of the crystallite (convention as in the monograph (Bunge, 1969)).

The correspondence between the Euler angles

$$
\left(\varphi_{1}, \phi, \varphi_{2}\right) \in[0,2 \pi] \times[0, \pi] \times[0,2 \pi]=\Omega
$$

and a point in the orientation space given by $\tilde{g}: \Omega \rightarrow \operatorname{SO}(3)$ is one-to-one unless $\phi=0$ or $\phi=\pi$. In such cases the rotation is determined by the sum $\varphi_{1}+\varphi_{2}$ or by the difference $\varphi_{1}-\varphi_{2}$, respectively. Thus to the pair $\left(\varphi_{1}, 0, \varphi_{2}\right),\left(\varphi_{1}^{\prime}, 0, \varphi_{2}^{\prime}\right)$ when $\varphi_{1}+$ $\varphi_{2}=\varphi_{1}^{\prime}+\varphi_{2}^{\prime}(\bmod 2 \pi)$ there corresponds the same rotation and they should be identified. In the same way the points $\tilde{g}\left(\varphi_{1}, \pi, \varphi_{2}\right)$ and $\tilde{g}\left(\varphi_{1}^{\prime}, \pi, \varphi_{2}^{\prime}\right)$ are identical if $\varphi_{1}-\varphi_{2}=\varphi_{1}^{\prime}-\varphi_{2}^{\prime}(\bmod 2 \pi)$.

There exists the possibility of choosing the parameters describing the orientation in such a way that the distinction of the direction $y$ is equivalent with the determination of two of them, while the third will be associated with the rotation around this direction. Using such coordinates the function $f$ in Eq. (1) may be written as depending explicitly on the integration variable, which will be represented by the third parameter, while the two first will determine the projection line.

After Pospiech (1980) we shall use the parameters $\alpha, \beta, \gamma$ with the above properties, which, have a simple geometrical meaning. Let $\theta$ be a polar coordinate and $\psi$ the azimuth of the vector $h$ in the crystallite coordinate system. The same vector in the sample coordinate system is denoted by $y$ and determined by the polar angle $\alpha$ and the azimuth angle $\beta$. Let, moreover, $K$ denote one of the cartesian coordinate systems such that the axis $Z$ of each of them has the direction and sense of the vector $y$.

It is easy to check that the rotation $\tilde{g}\left(\beta+\pi / 2, \alpha, \xi_{1}\right)$ of the sample coordinate system transforms this system into the system $K$, $\tilde{g}(\mu, 0, \xi)$ are rotations within the family of $K$ systems, and $\tilde{g}\left(\mu_{1}, \theta, \pi / 2-\psi\right)$ transforms $K$ into the crystallite coordinate 
system. The parameters $\mu, \mu_{1}, \xi, \xi_{1}$ are rotation angles about the same axis. Thus we may write

$$
\tilde{g}\left(\mu_{1}, \theta, \pi / 2-\psi\right) \tilde{g}(\mu, 0, \xi) \tilde{g}\left(\pi / 2+\beta, \alpha, \xi_{1}\right)=H(h) G(\gamma) Y(y)
$$

where

$$
\begin{aligned}
H(h) & :=g(0, \theta, \pi / 2-\psi), & G(\gamma) & :=g(\gamma, 0,0), \\
Y(y) & :=g(\pi / 2+\beta, \alpha, 0), & \gamma & :=\mu+\mu_{1}+\xi+\xi_{1} .
\end{aligned}
$$

The parameters $\alpha, \beta, \gamma$ are the new coordinates in the orientation space. The relation between them and the angles $\varphi_{1}, \phi, \varphi_{2}$

$$
\tilde{g}\left(\varphi_{1}, \phi, \varphi_{2}\right)=H(h) G(\gamma) Y(y)
$$

is simultaneously an equation of the projection line with the parameter $\gamma$ in the space of Euler angles. The set of orientations $\{g \in \mathrm{SO}(3): g=H(h) G(\gamma) Y(y), \gamma \in[0,2 \pi[\}$ represents the solution of Eq. (2) in the space of proper rotations. The parameters $\theta, \psi$ determine the family of the projection lines. A single line of this family is uniquely determined by a pair of the coordinates $\alpha, \beta$. To each of the sets of pairs $\{(\alpha, \beta): \alpha=0, \quad \beta \in[0,2 \pi[\}$ and $\{(\alpha, \beta): \alpha=\pi, \beta \in[0,2 \pi[\}$ corresponds one projection line. (As an example a projection line $[h, y]$ for $h \equiv\{\theta=\pi / 2, \psi=\pi / 4\} \equiv(110)$ and for $y \equiv\{\alpha=3 \pi / 4, \beta=0\}$ is shown on Figures $1 \mathrm{a}, \mathrm{b}$.)

From a comparison of the elements of the matrices on the left and on the right side of Eq. (4) one obtains the following dependence of the parameters $\varphi_{1}, \phi, \varphi_{2}$ on $\alpha, \beta, \gamma$

$$
\begin{aligned}
\cos \phi= & -\sin \theta \cos \gamma \sin \alpha+\cos \theta \cos \alpha, \\
\cos \varphi_{1}= & -(\sin \theta \sin \gamma \cos \beta+\sin \theta \cos \gamma \sin \beta \cos \alpha+ \\
& +\cos \theta \sin \beta \sin \alpha) / \sin \phi \\
\sin \varphi_{1}= & (-\sin \theta \sin \gamma \sin \beta+\sin \theta \cos \gamma \cos \beta \cos \alpha+ \\
& +\cos \theta \cos \beta \sin \alpha) / \sin \phi, \\
\cos \varphi_{2}= & (-\cos \psi \sin \gamma \sin \alpha+\sin \psi \cos \theta \cos \gamma \sin \alpha+ \\
& +\sin \psi \sin \theta \cos \alpha) / \sin \phi \\
\sin \varphi_{2}= & (\sin \psi \sin \gamma \sin \alpha+\cos \psi \cos \theta \cos \gamma \sin \alpha+ \\
& +\cos \psi \sin \theta \cos \alpha) / \sin \phi
\end{aligned}
$$

when $\phi \neq 0$, $\pi$. 


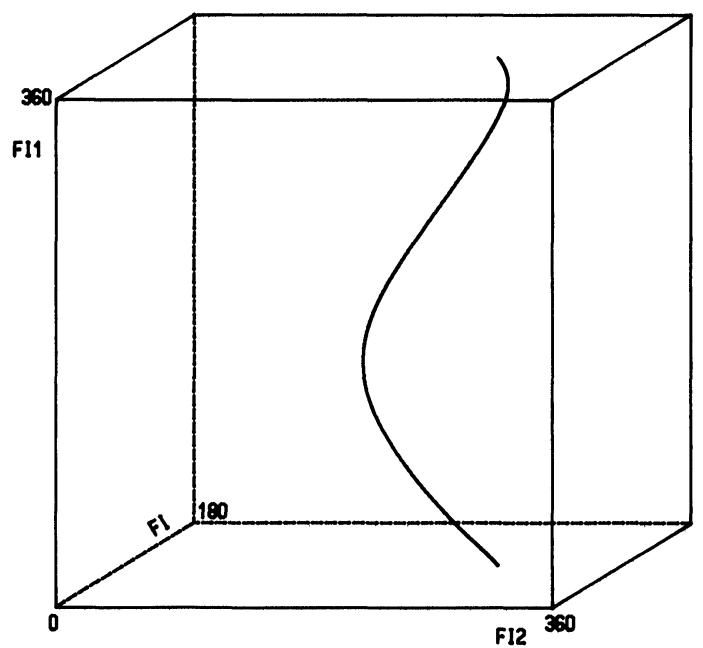

a)

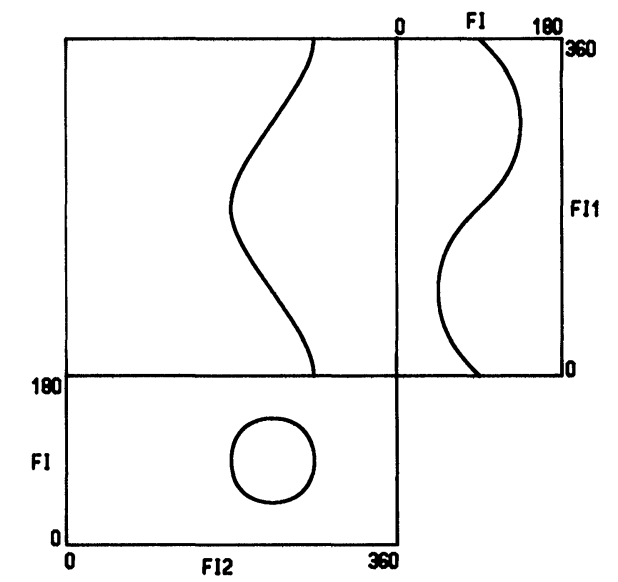

b)

Figure 1. a) The projection line $[h, y]$ in the space of Euler angles for $h \equiv\left\{\theta=90^{\circ}\right.$, $\left.\psi=45^{\circ}\right\} \equiv(110)$ and for $y \equiv\left\{\alpha=135^{\circ}, \beta=0^{\circ}\right\}$. b) The line from Fig. 1a projected on planes perpendicular to the axes $\varphi_{1}, \phi, \varphi_{2}$. 
When $\phi=0, \pi$ then, respectively,

$$
\begin{aligned}
& \cos \left(\varphi_{1}+\varphi_{2}\right), \cos \left(\varphi_{1}-\varphi_{2}\right)= \\
& =-\sin \psi \cos \gamma \sin \beta+\cos \psi \cos \theta \sin \gamma \sin \beta+ \\
& \quad-\sin \psi \sin \gamma \cos \beta \cos \alpha-\cos \psi \cos \theta \cos \gamma \cos \beta \cos \alpha+ \\
& \quad+\cos \psi \sin \theta \cos \beta \sin \alpha, \\
& \sin \left(\varphi_{1}+\varphi_{2}\right), \sin \left(\varphi_{1}-\varphi_{2}\right)=\sin \psi \cos \gamma \cos \beta+ \\
& \quad-\cos \psi \cos \theta \sin \gamma \cos \beta-\sin \psi \sin \gamma \sin \beta \cos \alpha+ \\
& \quad-\cos \psi \cos \theta \cos \gamma \sin \beta \cos \alpha+\cos \psi \sin \theta \sin \beta \sin \alpha .
\end{aligned}
$$

Reversed relations are found from $G(\gamma) Y(y)=H^{-1}(h) g\left(\varphi_{1}, \phi, \varphi_{2}\right)$ $\cos \alpha=\sin \theta \sin \left(\varphi_{2}+\psi\right) \sin \phi+\cos \theta \cos \phi$, $\cos \beta=\left(\sin \theta \cos \varphi_{1} \cos \left(\varphi_{2}+\psi\right)-\sin \theta \sin \varphi_{1} \sin \left(\varphi_{2}+\psi\right) \cos \phi+\right.$

$$
\left.+\cos \theta \sin \varphi_{1} \sin \phi\right) / \sin \alpha,
$$

$\sin \beta=\left(\sin \theta \sin \varphi_{1} \cos \left(\varphi_{2}+\psi\right)+\right.$

$\left.+\sin \theta \cos \varphi_{1} \sin \left(\varphi_{2}+\psi\right) \cos \phi-\cos \theta \cos \varphi_{1} \sin \phi\right) / \sin \alpha$,

$\cos \gamma=\left(\cos \theta \sin \left(\varphi_{2}+\psi\right) \sin \phi-\sin \theta \cos \phi\right) / \sin \alpha$,

$\sin \gamma=-\cos \left(\varphi_{2}+\psi\right) \sin \phi / \sin \alpha$,

when $\alpha \neq 0, \pi$.

When $\alpha=0, \pi$ then, respectively,

$$
\begin{aligned}
\cos (\beta+\gamma), \cos (\beta-\gamma)= & \sin \varphi_{1} \sin \left(\varphi_{2}+\psi\right)- \\
& -\cos \varphi_{1} \cos \left(\varphi_{2}+\psi\right) \cos \phi, \\
\sin (\beta+\gamma), \sin (\beta-\gamma)= & -\cos \varphi_{1} \sin \left(\varphi_{2}+\psi\right)- \\
& -\sin \varphi_{1} \cos \left(\varphi_{2}+\psi\right) \cos \phi .
\end{aligned}
$$

We shall give two basic properties of the projection lines in the space of Euler angles $\Omega$ :

(i) Lines of the same family ( $h=$ const) do not intersect. To each three angles $\left(\varphi_{1}, \phi, \varphi_{2}\right)$ a rotation is ascribed uniquely, and to each rotation there is ascribed a pair $(\alpha \neq 0, \pi, \beta)$ or sets of pairs $\{(\alpha, \beta): \alpha=0, \beta \in[0,2 \pi[\}$ or $\{(\alpha, \beta): \alpha=\pi, \beta \in[0,2 \pi[\}$, each of which represents a single line. Intersection does not occur for $\phi=0, \pi$, either, in spite of the above mentioned identifications. In these cases the parameters of the lines depend only on the sum or 
on the difference of the angles $\varphi_{1}, \varphi_{2}$, respectively since we have

$$
\alpha=\theta, \quad \beta=\varphi_{1}+\varphi_{2}+\psi, \quad \gamma=\pi \text { when } \phi=0
$$

and

$$
\alpha=\pi-\theta, \quad \beta=\varphi_{1}-\varphi_{2}-\psi, \quad \gamma=0 \text { when } \phi=\pi .
$$

(ii) A family of projection lines fills up the rotation space. For each point $\left(\varphi_{1}, \phi, \varphi_{2}\right)$ it is possible, using (6), to find the parameters $\alpha, \beta$ of a line containing it.

\section{THE INFLUENCE OF THE SYMMETRY ON THE GEOMETRY OF PROJECTION LINE}

We shall consider now which new aspects are introduced to the description of the projection lines by taking into account the crystal and sample symmetries.

Let $C$ and $S$ denote the groups of symmetry of the crystal and of the sample, respectively and both are finite subgroups of the group $\mathrm{O}(3)$. The points $g$ and $g^{\prime}$ of the orientation space $\mathrm{SO}(3)$ will be defined as symmetrically equivalent if there exist such elements $c \in C$ and $s \in S$, that $g^{\prime}=c^{-1} g s$. The representatives of all sets of symmetrically equivalent points selected in the space of proper rotations make an area which is symmetrically equivalent to the space (in brief-symmetrically equivalent area (SEA)). The ODF reduced to SEA contains the same amount of information as the ODF in the whole orientation space.

With the symmetry groups $C$ and $S$ established we shall define the family $H$ of the mappings $h_{c, s}: \mathrm{SO}(3) \rightarrow \mathrm{SO}(3)$

$$
h_{c, s}: g \rightarrow c^{-1} g s, \quad c \in C, \quad s \in S .
$$

For the image $h_{c, s}(g)$ to be the proper rotation for $g \in \mathrm{SO}(3), c$ and $s$ must be simultaneously either proper or improper elements of symmetry.

The above considerations may be put briefly: the symmetries of the crystal and of the sample imply the existence of a group of mappings of the space of proper rotations, with respect to which the ODF is invariant $\left(f(g)=f\left(h_{c, g}(g)\right)\right.$ for arbitrary $h_{c, s} \in H$ and $g \in$ $\mathrm{SO}(3))$. 
The existence of symmetrically equivalent points and the possibility of separating a symmetrically equivalent area have their reflection in the space of Euler angles. The transformations $h_{c, s}$ induce in it the mappings $H_{c, s}: \Omega \rightarrow \Omega$ defined as

$$
h_{c, s}\left(\tilde{g}\left(\varphi_{1}, \phi, \varphi_{2}\right)\right)=\tilde{g}\left(H_{c, s}\left(\varphi_{1}, \phi, \varphi_{2}\right)\right) \text {. }
$$

A detailed discussion of them can be found in the paper by Pospiech et al. (1974). These mappings enable to represent symmetrically equivalent lines in the space of Euler angles.

Let us concentrate again on the projection lines. An image of the line $[h, y]$ in the mapping $h_{c, s}$ is, as it follows from (2), the line sy from the family $c h: h_{c, g}([h, y])=[c h, s y]$. Taking the sum of images of the line $[h, y]$ in all the mappings from $H$ we shall obtain a set of all projection lines symmetrically equivalent to the line $[h, y]$. Instead of considering the whole set we can restrict our considerations to that part of it which is contained in a symmetrically equivalent area. This subset will be denoted by $[[h, y]]$ and termed a line reduced to the symmetrically equivalent area

$$
[[h, y]]=\operatorname{SEA} \cap \bigcup_{H} h_{c, s}([h, y]) .
$$

(Summation over all elements of the group $H$.)

The line $[[h, y]]$ is identical with the line $\left[\left[c^{\prime} h, s^{\prime} y\right]\right]$ for all $c^{\prime} \in C$ and $s^{\prime} \in S$ satisfying the condition $\operatorname{det}\left(c^{\prime} s^{\prime}\right)=+1$, as we have $\left[c^{\prime} h, s^{\prime} y\right]=h_{c^{\prime}, s^{\prime}}([h, y])$. And since, as it has been mentioned, $H$ is a group, hence

$$
\bigcup_{H} h_{c, s}\left(h_{c^{\prime}, s^{\prime}}([h, y])\right)=\bigcup_{H} h_{c, s}([h, y]) .
$$

Lines of the same family $h$ reduced to the symmetrically equivalent area (i.e. lines $[[h, y]]$ with established $h$, and $y$ changing over its whole range) intersect. Through a point of the symmetrically equivalent area there pass exactly as many such lines as there are elements in the set of symmetrically equivalent points represented by this point. It should also be considered here that a symmetry operation may lead to overlapping of projection lines. (Figures 2a,b show the geometry of lines of the type $\{111\}$ (for cubic crystal symmetry) intersecting in one point of the Euler angles space.) 


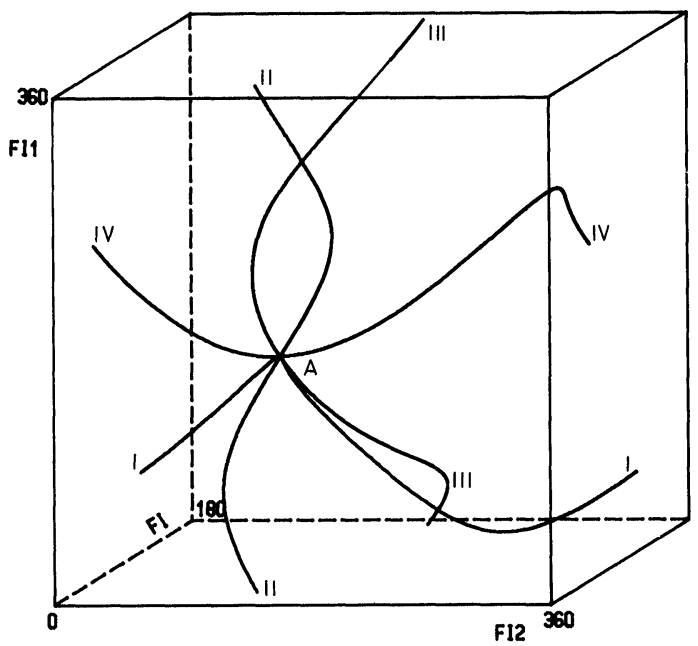

a)

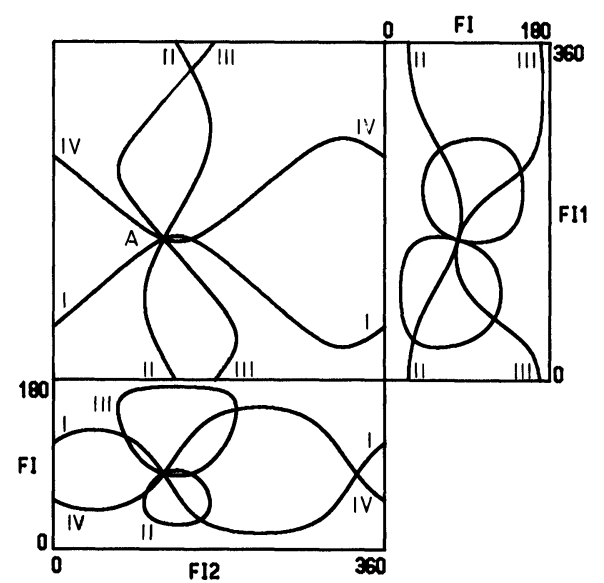

b)

Figure 2. a) Projection lines in the space of Euler angles for symmetrically equivalent vectors $h$ of the type $\{111\}$ (for cubic crystal symmetry) which intersect at a point indicated by $A$. The symbols I, II, III and IV indicate (111), (1i11), (1111) and (īi), respectively. b) The lines from Fig. 2a projected on planes perpendicular to the axes $\varphi_{1}, \phi, \varphi_{2}$. 
The overlapping of lines when they are reduced to the symmetrically equivalent area is subject to certain regularities. Following statements hold:

1. If for a certain element $c$ of the crystal symmetry there exists a point $g$ in the rotation space lying on the line $[h, y]$, such that the point $h_{c, e}(g)$ symmetrically equivalent to $g$ lies also on the same line, then for each point $g^{\prime}$ of an arbitrary line $\left[h, y^{\prime}\right]$ of this family the point $h_{c, e}\left(g^{\prime}\right)$ also lies on the line $\left[h, y^{\prime}\right]$.

In other words, if due to the existence of a certain crystal symmetry two points of the same line overlap, then the other points of this lines overlap in pairs and, moreover, this overlapping (within one line) takes place for all lines of this family.

2 . If there exists an element $s$ of the sample symmetry and a point $g$ of the projection line $[h, y]$ such that $h_{e, s}(g)$ also belongs to $[h, y]$, then with each point $g^{\prime}$ of the same line $[h, y]$ the point $h_{e, s}\left(g^{\prime}\right)$ belongs to this line and this takes place for fixed $y$ in any arbitrary family of lines.

Moreover, the following two statements of a slightly different character, are valid:

3. If for a certain point $g$ of the projection line $[h, y]$ and for an element $s$ of the sample symmetry the point $h_{e, s}(g)$ belongs to the line $\left[h, y^{\prime}\right]$, then for an arbitrary $g^{\prime}$ from $[h, y], h_{e, s}\left(g^{\prime}\right)$ is a point of $\left[h, y^{\prime}\right]$.

In other words, the symmetry of the sample does not lead to the intersection of projection lines of the same family when they are reduced to the symmetrically equivalent area, but only to overlapping of entire lines.

Analogously, there holds

4. If for a certain $g$ of the line $[h, y]$ and for an element $c$ of the crystal symmetry, $h_{c, e}(g)$ belongs to $\left[h^{\prime}, y\right]$, then for an arbitrary $g^{\prime}$ from the line $[h, y], h_{c, e}\left(g^{\prime}\right)$ is a point of the line $\left[h^{\prime}, y\right]$.

It should be added that the thesis of statement 1 is equivalent to the existence of a rotation axis parallel to vector $h$ in the group $C$.

Strictly speaking we have the next statement.

5. For established $h$ and $c \in C \cap \operatorname{SO}(3)$, the existence of an orientation $g \in[h, y]$ such that $c^{-1} g \in[h, y]$ leads to the relation $c h=h$. Conversely, if there occurs $c h=h$ then for arbitrary $g \in[h, y]$ also $c^{-1} g$ belongs to $[h, y]$. 


\section{PROJECTION LINES RELATED WITH POLE FIGURES}

As it is known, in normal experimental practice, not the projection but the pole figure $P_{h}(y)$ is obtained (Matthies, 1979):

$$
P_{h}(y)=\frac{1}{2}\left(P_{h}^{0}(y)+P_{-h}^{0}(y)\right)=\frac{1}{2}\left(P_{h}^{0}(y)+P_{h}^{0}(-y)\right) .
$$

The form of this expression suggests an analysis of the relation between points of the associated lines, i.e. the ones out of which one is determined by $y$ and the other one by $-y$, both of the same $h$.

The equality $P_{-h}^{0}(y)=P_{h}^{0}(-y)$ follows from the identity of the projection lines $[-h, y]$ and $[h,-y]$. This in turn follows directly from (2). According to (4) the point having a parameter $\gamma$ on the line $[-h, y]$ has the parameter $-\gamma$ on $[h,-y]$.

The question arises what are the symmetry conditions at which for established $h$ the lines $[[h, y]]$ and $[[h,-y]]$ are identical. The answer can be formulated in the following way:

6. The existence of the element $c$ of the crystal symmetry, such that $c h=-h$ and $c \in \mathrm{SO}(3)$ is a necessary and sufficient condition for the lines $[[h, y]]$ and $[[h,-y]]$ to be identical for each $y$.

Example. Certain, exceptionally simple dependences between the points of the associated lines are connected with the above statement. On the basis of the equations (4) and

$$
g\left(\varphi_{1}^{\prime}, \phi^{\prime}, \varphi_{2}^{\prime}\right)=H(h) G\left(\gamma_{1}\right) Y(-y)
$$

and utilizing the fact that $Y(-y)=Q Y(y)$ and $H(-h)=H(h) Q$, where $Q:=g(0, \pi, \pi)$ we get

$$
g\left(\varphi_{1}^{\prime}, \phi^{\prime}, \varphi_{2}^{\prime}\right)=H(-h) G\left(-\gamma-\gamma_{1}\right) H^{-1}(h) g\left(\varphi_{1}, \phi, \varphi_{2}\right) .
$$

The matrix $H(-h) G\left(-\gamma-\gamma_{1}\right) H^{-1}(h)$ has a particularly simple form and its elements do not depend on the angle $\theta$ when $\gamma_{1}=\pi-\gamma$. One gets (see the appendix)

$$
\begin{aligned}
g\left(\varphi_{1}^{\prime}, \phi^{\prime}, \varphi_{2}^{\prime}\right) & =g(2 \psi, \pi, \pi) g\left(\varphi_{1}, \phi, \varphi_{2}\right) \\
& =g\left(\pi+\varphi_{1}, \pi-\phi,-2 \psi-\varphi_{2}\right) .
\end{aligned}
$$

The coordinates $\varphi_{1}, \phi, \varphi_{2}$ of the point $\gamma$ on the projection line $[h, y]$ are thus connected with the coordinates $\varphi_{1}^{\prime}, \phi^{\prime}, \varphi_{2}^{\prime}$ of the 
point $\gamma_{1}=\pi-\gamma$ on the associated line $[h,-y]$ by the following formulae (Pospiech et al., 1984):

$$
\varphi_{1}^{\prime}=\pi+\varphi_{1}, \quad \phi^{\prime}=\pi-\phi, \quad \varphi_{2}^{\prime}=-2 \psi-\varphi_{2} .
$$

Figures 3a,b show an example of associated lines.

Let the two-fold axis $L_{y}^{2}$ exist as an element of the crystal symmetry. The mapping $H_{L y, e}^{2}$ has the form $H_{L y, e}^{2}\left(\varphi_{1}, \phi, \varphi_{2}\right)=$ $\left(\pi+\varphi_{1}, \pi-\phi,-\varphi_{2}\right)$. The points $g\left(\varphi_{1}, \phi, \varphi_{2}\right)$ of the line $[h, y]$ and $g\left(\varphi_{1}^{\prime}, \phi^{\prime}, \varphi_{2}^{\prime}\right)$ of the line $[h,-y]$ are thus symmetrically equivalent if $\psi=0$ or $\psi=\pi$. This follows also directly from our earlier statement 6 , since $L_{y}^{2} h=-h$ if the vector $h$ lies in the plane $X-Z$ of the Carthesian coordinate system, i.e. just when $\psi=0$ or $\psi=\pi$. Hence if a pole figure is determined by the vector $h$ such that $\psi=0$, $\pi$ and the axis $L_{y}^{2}$ is an element of the crystal symmetry, this figure is identical with the projection $P_{h}^{0}$.

The sources of the existing ambiguity of the solution of the ODF reproduction problem are being found in the specific way of intersection of integral lines (Matthies, 1980, Matthies, 1981). The statements given below, explain exhaustively for which lines and at which points the intersection occurs.

Of basic importance is the fact that

7. For arbitrary $h, h^{\prime} \neq \pm h, y, y^{\prime}$ the lines $[h, y]$ and $\left[h^{\prime}, y^{\prime}\right]$ intersect at one point at most.

However, we have the next statement, important because of (10)

8. For arbitrary $h, h^{\prime} \neq \pm h, y, y^{\prime}$ if the lines $[h, y]$ and $\left[h^{\prime}, y^{\prime}\right]$ have a common point, then also associated lines $[h,-y]$ and $\left[h^{\prime},-y^{\prime}\right]$ intersect.

When the parameter $\gamma$ of the point of intersection on the line $[h, y]$ is established, one can find, using first Eqs. (5) and then Eqs. (6), the parameters $\alpha^{\prime}, \beta^{\prime}, \gamma^{\prime}$ of that point on the line of the family $h^{\prime}$. There arises the question concerning the coordinates of the point of intersection of associated lines.

9. If the point common to the lines $[h, y]$ and $\left[h^{\prime}, y^{\prime}\right]\left(h^{\prime} \neq \pm h\right)$ has the parameter $\gamma$ on the line $[h, y]$, the point of intersection of associated lines has the parameter $\gamma_{1}=\gamma_{0}-\gamma(\bmod 2 \pi)$, where $\gamma_{0}$ is given in the following way

$$
\sin \gamma_{0}=\frac{-2 a_{1} a_{2}}{a_{1}^{2}+a_{2}^{2}}, \quad \cos \gamma_{0}=\frac{a_{1}^{2}-a_{2}^{2}}{a_{1}^{2}+a_{2}^{2}}
$$


a)

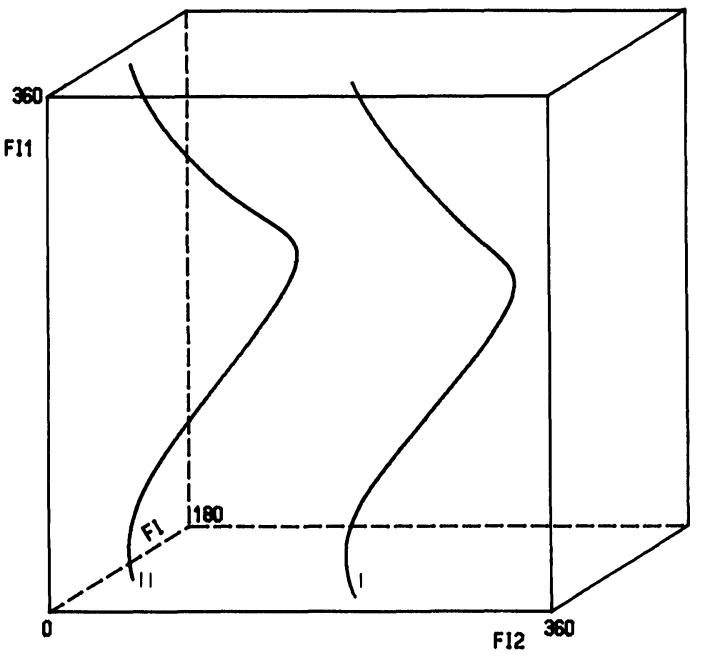

b)

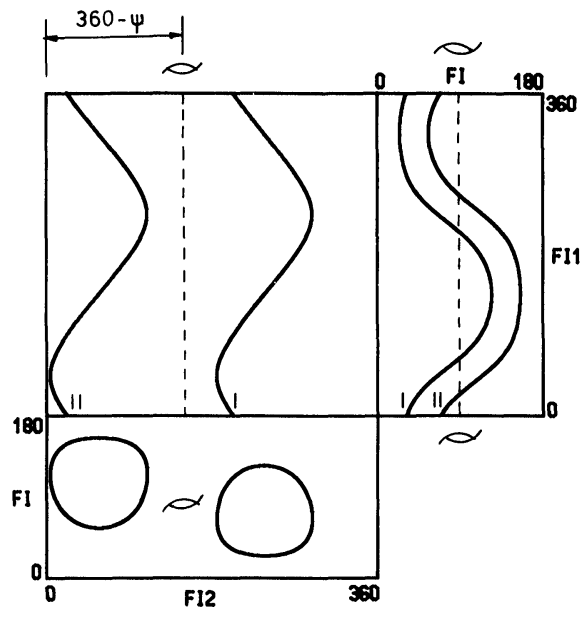

Figure 3. a) Associated projection lines $[h, y]$ and $[h,-y]$ in the space of Euler angles for $h \equiv\left\{\theta=74.5^{\circ}, \psi=213.7^{\circ}\right\} \equiv(\overline{3} 21)$ for $y \equiv\left\{\alpha=50^{\circ}, \beta=45^{\circ}\right\}$ and $y \equiv\left\{180^{\circ}-\alpha=130^{\circ}, 180^{\circ}+\beta=225^{\circ}\right\}$ (marked by I and II, respectively). b) The lines from Fig. 3a projected on planes perpendicular to the axes $\varphi_{1}, \phi, \varphi_{2}$. The lines are related by a two-fold screw axis (with the translation $\Delta \varphi_{1}=180^{\circ}$ ) according to Eq. (14). 
and

$$
\begin{aligned}
& a_{1}=\sin \theta^{\prime} \sin \left(\psi-\psi^{\prime}\right), \\
& a_{2}=\sin \theta^{\prime} \cos \theta \cos \left(\psi-\psi^{\prime}\right)-\cos \theta^{\prime} \sin \theta
\end{aligned}
$$

It follows from the above, that if three lines of different families intersect at one point, the lines associated to them intersect, in general, at different points. It is explained in statement 10 .

10. Let lines of three different families $h, h^{\prime}, h^{\prime \prime}\left(h^{\prime} \neq \pm h\right.$, $\pm h \neq h^{\prime \prime} \neq \pm h^{\prime}$ ) have a common point. The adequate associated lines intersect also at one point if and only if the vectors $h, h^{\prime}, h^{\prime \prime}$ are coplanar.

Figures $4 a, b$ show an example.

From the viewpoint of ambiguity in the ODF reproduction problem it is important also whether the given line $[h, y]$ can intersect a line $\left[h^{\prime}, y^{\prime}\right]$ and the associated line $\left[h^{\prime},-y^{\prime}\right]$ simultaneously. One can prove the following statement.

11. Let $[h, y]$ and $\left[h^{\prime}, y^{\prime}\right]\left(h^{\prime} \neq \pm h\right)$ have a common point. The same line $[h, y]$ intersects the line $\left[h^{\prime},-y^{\prime}\right]$ if and only if the vector $h$ is perpendicular to the vector $h^{\prime}$.

It is obvious (from st. 11 or 8 ) that if $[h, y]$ intersects $\left[h^{\prime}, y^{\prime}\right]$ as well as $\left[h^{\prime},-y^{\prime}\right]$, then also $[h,-y]$ intersects both these lines. An example is illustrated on Figs: $5 a, b$.

Finally, for the sake of completeness we give the statement 12 .

12. Parameters $\gamma, \gamma_{2}$ on $[h, y]$ of the points of intersection of this line by $\left[h^{\prime}, y^{\prime}\right]$ and $\left[h^{\prime},-y^{\prime}\right]$, respectively, are related by $\gamma_{2}=\gamma+\pi$ $(\bmod 2 \pi)$.

The problem of the influence of the symmetry should be considered. Due to symmetry the points of ambiguity (i.e. such points as those described in statements 8 and 11) can be symmetrically equivalent. It appears to be the simplest way to consider these symmetrical equivalences separately for each individual cases of symmetry.

In the Example given above, parameters of symmetrically equivalent points of associated lines fulfil the condition $\gamma^{\prime}+\gamma=\pi$. Using Eqns. (15) and Eqns. (16) we obtain that $\gamma_{0}=\gamma_{1}+\gamma=\pi$ when $\psi^{\prime}=\psi+k \pi$ or $\theta^{\prime}=0, \pi$. When $\theta=0, \pi$ the relation $\gamma_{1}+\gamma=\pi$ leads to $\psi^{\prime}=0, \pi$. Therefore, in this case the points described in statement 8 are symmetrically equivalent when not only vector $h$ but also vector $h^{\prime}$ belong to the $X Z$-plane. 
a)

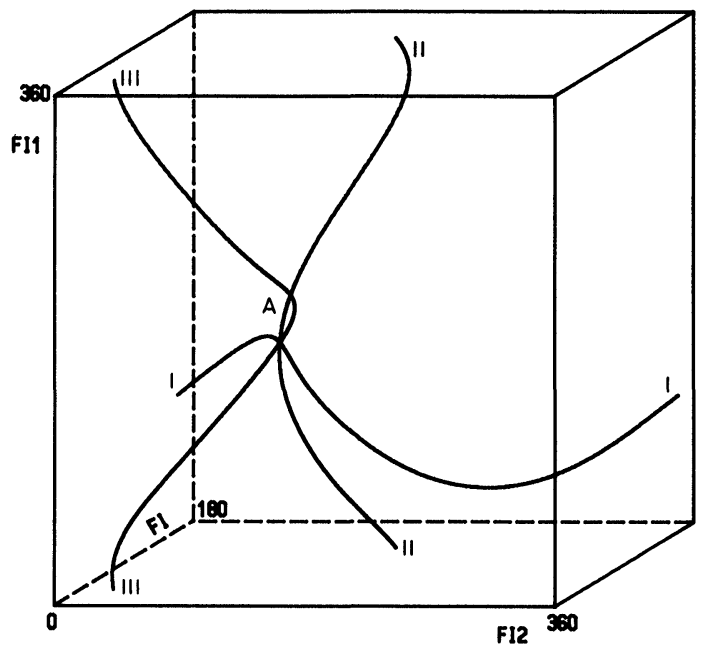

b)

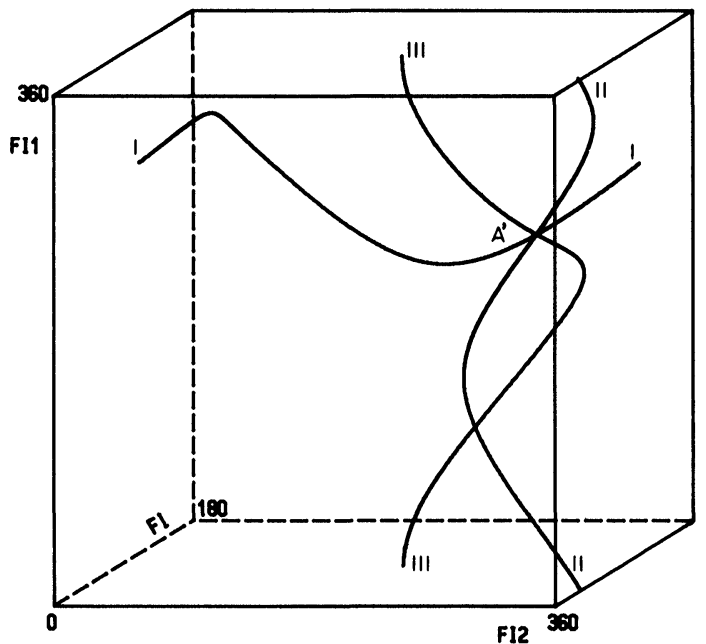

Figure 4. a) Projection lines belong to three coplanar vectors $h \equiv\left\{\theta=45^{\circ}\right.$, $\left.\psi=90^{\circ}\right\} \equiv(011), h^{\prime} \equiv\left\{\theta^{\prime}=54.7^{\circ}, \psi^{\prime}=135^{\circ}\right\} \equiv(\overline{1} 11)$ and $h^{\prime \prime} \equiv\left\{\theta^{\prime \prime}=72.4^{\circ}, \quad \psi^{\prime \prime}=\right.$ $\left.18.4^{\circ}\right\} \equiv(311)$ (marked by I, II and III, respectively) which intersect at one point $A$ $\left(\varphi_{1}=146.5^{\circ}, \phi=119.5^{\circ}, \varphi_{2}=95.7^{\circ}\right), y \equiv\left\{\alpha=114.2^{\circ}, \beta=6^{\circ}\right\}, y^{\prime} \equiv\left\{\alpha^{\prime}=146.6^{\circ}\right.$, $\left.\beta^{\prime}=346.8^{\circ}\right\}$ and $y^{\prime \prime} \equiv\left\{\alpha^{\prime \prime}=52.5^{\circ}, \beta^{\prime \prime}=27.0^{\circ}\right\}$. b) The geometry of the projection lines associated with the lines from Fig. 4a. They intersect also at one point (indicated by $A^{\prime}\left(\varphi_{1}=233.7^{\circ}, \phi=85,0^{\circ}, \varphi_{2}=299.6^{\circ}\right)$ ). 
a)

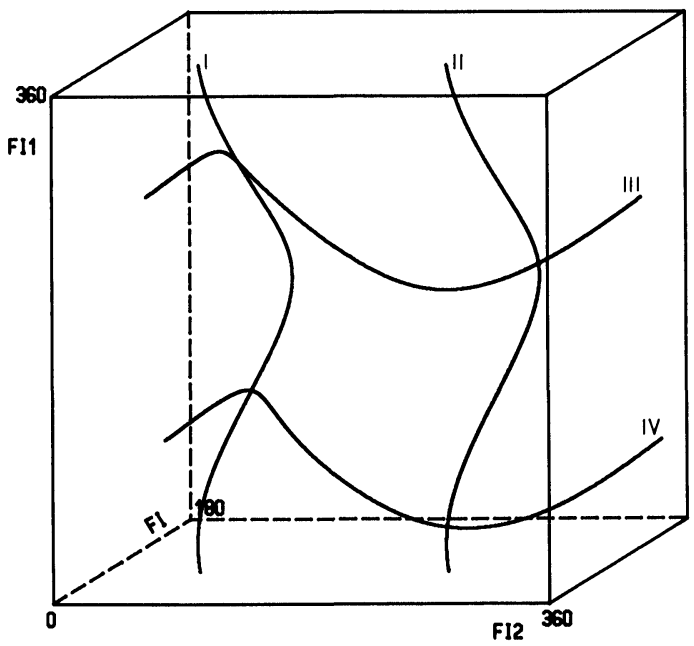

b)

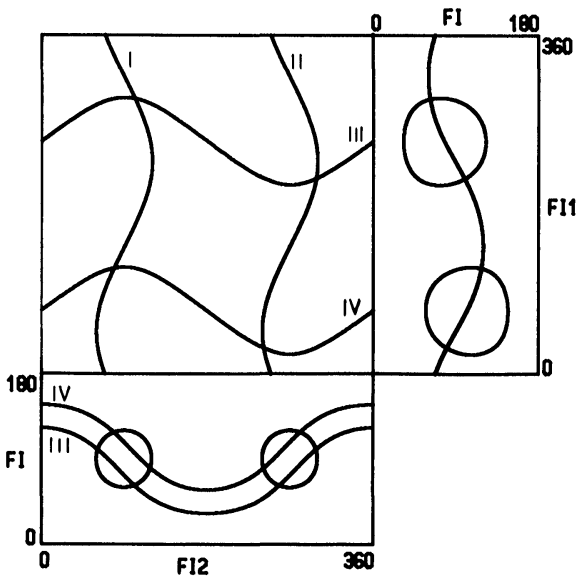

Figure 5. a) The intersection geometry of associated lines $\mathrm{I}-[h, y]$ and $\mathrm{II}-[h,-y]$ and of associated lines III- $\left[h^{\prime}, y^{\prime}\right]$ and IV $-\left[h^{\prime},-y^{\prime}\right]$, when the vectors $h$ and $h^{\prime}$ are perpendicular, $h \equiv\left\{\theta=90^{\circ}, \psi=0^{\circ}\right\} \equiv(100), y \equiv\left\{\alpha=30^{\circ}, \beta=45^{\circ}\right\}, h^{\prime} \equiv\left\{\theta=45^{\circ}\right.$, $\left.\psi=90^{\circ}\right\} \equiv(011), y^{\prime} \equiv\left\{\alpha=77.8^{\circ}, \beta=157^{\circ}\right\}$. b) The lines from Figure 5a projected on planes perpendicular to the axes $\varphi_{1}, \phi, \varphi_{2}$. 
One can (in general) show that the symmetrical equivalence of points described in statement 8 is equivalent to the existence of two-fold rotation axis perpendicular to the vectors $h$ and $h^{\prime}$.

Similarly the points described in statement 11 are symmetrically equivalent if and only if the group of crystal symmetry contains a two-fold rotation axis parallel to the vector $h$ and a two-fold rotation axis parallel to the vector $h^{\prime}$.

\section{FINAL REMARKS}

The knowledge of the properties of the projection lines in the ODF space is interesting particularly in problems of the ODF approximation by direct methods. In these methods the ODF approximation problem is solved through discretisation of the pole figure area and the ODF space on the basis of the system of equations $p_{k}=\sum_{j} f_{j} R_{j k}$ in which the matrix $R$ is given by the geometry of the projection lines (which have in this case the form of tubes (see e.g. Pawlik, 1986)). Due to the centrosymmetry of the pole figure the solution is ambiguous. On the other hand it is not yet fully clarified how the properties of the equation system are influenced by the kind of the geometry of the projection lines, e.g. by the choice of positions of the vectors $h$, by crystal symmetries and by the number of various vectors $h$ (number of pole figures) with a given relation between the resolution of the pole figure and the resolution of the solution (Pospiech, 1984).

\section{References}

1. Bunge, H. J. (1969) Mathematische Methoden der Texturanalyse, AkademieVerlag, Berlin.

2. Esling, C., Bunge, H. J. and Muller, J. (1980) Journ. Phys. Lett. 41, 543.

3. Matthies, S. (1979) Phys. Stat. Sol. (b) 92, K135.

4. Matthies, S. (1980) Kristall u. Technik 15, 431.

5. Matthies, S., Crystal Research and Technology 16, 513.

6. Pawlik, K., (1986) Phys. Stat. Sol., (b) 134, 477.

7. Pospiech, J., Gnatek, A. and Fichtner, K., (1974) Kristall u. Technik 9, 729.

8. Pospiech, J. (1980) Kristall u. Technik 15, 1481.

9. Pospiech, J., Romańska, M. and Lücke, K., Proc. ICOTOM 7, Noordwijkerhout 1984, p. 823.

10. Pospiech, J., Proc. ICOTOM 7, Noordwijkerhout 1984, Supplement p. 13. 


\section{APPENDIX}

We will give outlines of proofs of the statements included in the text but first we will derive the relations (12)-(14).

The following equalities will be useful (They are a direct result of the definition of Euler angles and their properties given in the first part of the text.):

a) $\tilde{g}(\mu, v, \xi)=\tilde{g}(\xi, 0,0) \tilde{g}(0, v, 0) \tilde{g}(0,0, \mu)$,

b) $\tilde{g}\left(\mu_{1}, v_{1}, \xi_{1}\right) \tilde{g}\left(\mu_{2}, v_{2}, \xi_{2}\right)=\tilde{g}\left(\mu_{1}+\xi_{2}, v_{1}, \xi_{1}\right) \tilde{g}\left(\mu_{2}, v_{2}, 0\right)$ $=\tilde{g}\left(0, v_{1}, \xi_{1}\right) \tilde{g}\left(\mu_{2}, v_{2}, \xi_{2}+\mu_{1}\right)$,

c) $\tilde{g}\left(0, v_{1}, 0\right) \tilde{g}\left(0, v_{2}, 0\right)=\tilde{g}\left(0, v_{1}+v_{2}, 0\right)$,

d) $\tilde{g}(\mu, 0, v)=\tilde{g}(\mu+v, 0,0)=\tilde{g}(0,0, \mu+v)$

e) $\tilde{g}(\mu, \pi, v)=\tilde{g}(\mu-v, \pi, 0)=\tilde{g}(0, \pi, v-\mu)$

f) $\tilde{g}^{-1}(\mu, v, \xi)=\tilde{g}(-\xi,-v,-\mu)$,

g) $\tilde{g}(\mu, v, \xi)=\tilde{g}(\pi+\mu,-v, \pi+\xi)$,

h) $\tilde{g}(0, \pi, \pi)=\tilde{g}(\pi, \pi, 0)=: Q$.

Next one can show that

i) $\tilde{g}(\mu, 0,0) Q=Q \tilde{g}(-\mu, 0,0)$.

$$
\begin{aligned}
g(\mu, 0,0) Q & =\tilde{g}(\mu, 0,0) \tilde{g}(0, \pi, \pi)=\tilde{g}(0, \pi, \pi+\mu) \\
& =\tilde{g}(-\mu, \pi, \pi)=\tilde{g}(0, \pi, \pi) \tilde{g}(0,0,-\mu) \\
& =Q \tilde{g}(-\mu, 0,0) .
\end{aligned}
$$

Analogously

j) $\tilde{g}(0,0, \xi) Q=Q \tilde{g}(0,0,-\xi)$,

k) $\tilde{g}(0, v, 0) Q=Q \tilde{g}(0,-v, 0)$.

Then it is easy to prove

1) $Y(-y)=Q Y(y)$.

$$
\begin{aligned}
& Y(-y)=\tilde{g}(\beta+\pi+\pi / 2, \pi-\alpha, 0)=\tilde{g}(\beta+\pi / 2, \\
& \quad \alpha-\pi, \pi)=\tilde{g}(\pi,-\pi, 0) \tilde{g}(\beta+\pi / 2, \alpha, 0)=Q Y(y)
\end{aligned}
$$

And in a similar way

m) $H(-h)=H(h) Q$.

Using $i, l, m$ we can show (12)

$$
\begin{aligned}
H(h) G\left(\gamma_{1}\right) Y(-y)= & H(h) Q G\left(-\gamma_{1}\right) Y(y) \\
& =H(-h) G\left(-\gamma_{1}-\gamma\right) H^{-1}(h)(H(h) G(\gamma) Y(y)) .
\end{aligned}
$$


The proof of (13) is a little longer:

$$
\begin{aligned}
H( & -h) G(\pi) H^{-1}(h)=H(h) Q G(\pi) H^{-1}(h) \\
\quad & \tilde{g}(0, \theta,-\psi+\pi / 2) \tilde{g}(0, \pi, \pi) \tilde{g}(0,0, \pi) \tilde{g}(\psi-\pi / 2,-\theta, 0) \\
& =\tilde{g}(0, \theta,-\psi+\pi / 2) \tilde{g}(0, \pi, 0) \tilde{g}(\psi-\pi / 2,-\theta, 0) \\
& =\tilde{g}(0, \theta,-\psi+\pi / 2) \tilde{g}(\psi-\pi / 2, \pi-\theta, 0) \\
& =\tilde{g}(-\psi+\pi / 2,0,0) \tilde{g}(0, \theta, 0) \tilde{g}(0, \pi-\theta, 0) \tilde{g}(0,0, \psi-\pi / 2) \\
& =\tilde{g}(-\psi+\pi / 2,0,0) \tilde{g}(0, \pi, 0) \tilde{g}(0,0, \psi-\pi / 2) \\
& =\tilde{g}(\psi-\pi / 2, \pi,-\psi+\pi / 2)=\tilde{g}(2 \psi, \pi, \pi) .
\end{aligned}
$$

And then

$$
\begin{aligned}
& \tilde{g}(2 \psi, \pi, \pi) \tilde{g}\left(\varphi_{1}, \phi, \varphi_{2}\right)=\tilde{g}(0, \pi, \pi) \tilde{g}\left(2 \psi+\varphi_{2}, 0,0\right) \tilde{g}\left(\varphi_{1}, \phi, 0\right) \\
& \quad=\tilde{g}\left(-2 \psi-\varphi_{2}, 0,0\right) \tilde{g}(0,-\phi, 0) \tilde{g}(\pi, \pi, 0) \tilde{g}\left(0,0, \varphi_{1}\right) \\
& \quad=\tilde{g}\left(-2 \psi-\varphi_{2}, 0,0\right) \tilde{g}(0, \pi-\phi, 0) \tilde{g}\left(0,0, \pi+\varphi_{1}\right) \\
& \quad=\tilde{g}\left(\pi+\varphi_{1}, \pi-\phi,-2 \psi-\varphi_{2}\right) .
\end{aligned}
$$

The relations (14) follow directly from the above equality.

St. 1. From the assumption, points $g$ and $c^{-1} g$ lie on the same line $[h, y]$. One can write $g=H(h) G(\gamma) Y(y)$ and $c^{-1} g=$ $H(h) G\left(\gamma_{1}\right) Y(y)$. From this we have $c^{-1}=H(h) G\left(\gamma_{1}-\gamma\right) H^{-1}(h)$. If the point $g^{\prime}$ belongs to $\left[h, y^{\prime}\right]$, i.e. $g^{\prime}=H(h) G\left(\gamma^{\prime}\right) Y\left(y^{\prime}\right)$, then $c^{-1} g^{\prime}=H(h) G\left(\gamma_{1}-\gamma+\gamma^{\prime}\right) Y\left(y^{\prime}\right)$, so $c^{-1} g^{\prime}$ is also the point of $\left[h, y^{\prime}\right]$.

The truth of st. 2-4 can be shown in analogous way.

St.5. The proof taking into consideration of statement 1 is similar to the proof of statement 6 .

St. 6. If there exists the element $c$ of crystal symmetry, such that $c h=-h$ and $c \in \operatorname{SO}(3)$, then we have directly the equality

$$
[[h, y]]=[[c h, e y]]=[[-h, y]]=[[h,-y]] \text {. }
$$

It is a little more difficult to show that the identity of lines [ $[h, y]]$ and $[[h,-y]]$ (for all $y$ and established $h$ ) leads to the existence of $c \in C$ such that $c h=-h$ and $c$ is a proper rotation. From the equality $[[h, y]]=[[h,-y]]$ and the assumption that the groups $C$ and $S$ are finite, there follows the existence of the mapping $h_{c, s}$ such that for infinite number of $g \in[h, y], h_{c, s}(g)$ belongs to $[h,-y]$. 
Therefore, for all $g \in[h, y]$ we have $h_{c, s}(g) \in[h,-y]$ and then $h_{c, s}([h, y])=[h,-y]$. For each $y$ and each $g \in[h, y]$ the following equations occur

$$
\begin{gathered}
g=H(h) G(\gamma) Y(y), \quad h_{c, s}(g)=H(c h) G\left(\gamma^{\prime}\right) Y(s y), \\
h_{c, s}(g)=H(h) G\left(\gamma^{\prime \prime}\right) y(-y) .
\end{gathered}
$$

Comparing the two last ones we get a new one

$$
Q H^{-1}(h) H(c h)=G\left(-\gamma^{\prime \prime}\right) Y(y) Y^{-1}(s y) G\left(-\gamma^{\prime}\right) .
$$

Its left-hand side is constant. In order to have the right-hand side constant one has to put

$$
Y(y) Y^{-1}(s y)=G(\dot{\gamma}) \quad \text { or } \quad Y(y) Y^{-1}(s y)=Q G(\dot{\gamma}) .
$$

In the first case $Y(y)=G(\dot{\gamma}) Y(s y)$ and $H(c h)=H(-h) G\left(\dot{\gamma}-\gamma^{\prime \prime}-\right.$ $\gamma)$. (The coordinates of the vector $h$ make the third column of matrix $H(h)$ and also of the matrix $H(h) G(\gamma)$ whereas the coordinates of the vector $y$ form the third row of the matrix $Y(y)$ and $G(\gamma) Y(y)$ for arbitrary $\gamma$.) Then $c$ fulfills $c h=-h$. Moreover, for every $y, s y=y$; so $s=e$. From the constraint $\operatorname{det}(c s)=+1, c$ is the proper rotation. The second case leads to the relations $c h=h$ and $s y=-y$ for all $y$. Hence, $s=i$ and $c$ is the improper rotation and can be written as $c=\bar{c} i$. According to the primary assumption that $i \in C$, we get $\bar{c} \in C \cap \mathrm{SO}(3)$ and $\bar{c} h=-h$.

St. 7. When the two lines have more than one point common, one can write at least two sets of equations

$$
\begin{aligned}
& H(h) G\left(\gamma_{1}\right) Y(y)=H\left(h^{\prime}\right) G\left(\gamma_{1}^{\prime}\right) Y\left(y^{\prime}\right), \\
& H(h) G\left(\gamma_{2}\right) Y(y)=H\left(h^{\prime}\right) G\left(\gamma_{2}^{\prime}\right) Y\left(y^{\prime}\right) .
\end{aligned}
$$

Eliminating $Y\left(y^{\prime}\right) Y^{-1}(y)$ we get

$$
H^{-1}\left(h^{\prime}\right) H(h) G\left(\gamma_{1}-\gamma_{2}\right)\left(H^{-1}\left(h^{\prime}\right) H(h)\right)^{-1}=G\left(\gamma_{1}^{\prime}-\gamma_{2}^{\prime}\right) .
$$

Since the $Z$-axis is the axis of rotation for the elements of the form $G(\gamma)$, there is

$$
H^{-1}\left(h^{\prime}\right) H(h)\left[\begin{array}{l}
0 \\
0 \\
1
\end{array}\right]=\left[\begin{array}{c}
0 \\
0 \\
\pm 1
\end{array}\right] \text {. }
$$


Moreover, $\left(H^{-1}\left(h^{\prime}\right) H(h)\right)_{33}=h \cdot h^{\prime}($ a scalar product $)$. Therefore, $h \cdot h^{\prime}= \pm 1$. But $h$ and $h^{\prime}$ are unity vectors; so $h^{\prime}= \pm h$.

St. 8 The lines $[h, y]$ and $\left[h^{\prime}, y^{\prime}\right]$ intersect. Then

$$
H(h) G(\gamma) Y(y)=H\left(h^{\prime}\right) G\left(\gamma^{\prime}\right) Y\left(y^{\prime}\right) .
$$

For the intersection of the associated lines to occur, there must exist the parameters $\gamma_{1}, \gamma_{1}^{\prime}$ such that

$$
H(h) G\left(\gamma_{1}\right) Y(-y)=H\left(h^{\prime}\right) G\left(\gamma_{1}^{\prime}\right) Y\left(-y^{\prime}\right) .
$$

Eliminating in the above equations the dependence on $y$ and $y^{\prime}$ and then on $h$ and $h^{\prime}$ one gets

$$
\begin{gathered}
H^{-1}\left(h^{\prime}\right) H(h) G\left(\gamma_{1}+\gamma\right)\left(H^{-1}\left(h^{\prime}\right) H(h)\right)^{-1}=G\left(\gamma_{1}^{\prime}+\gamma^{\prime}\right) Q \\
Y\left(y^{\prime}\right) Y^{-1}(y) G\left(\gamma_{1}-\gamma\right)\left(Y\left(y^{\prime}\right) Y^{-1}(y)\right)^{-1}=G\left(\gamma_{1}^{\prime}-\gamma^{\prime}\right) Q
\end{gathered}
$$

The parameters $\gamma_{1}, \gamma_{1}^{\prime}$ have to satisfy Eqs. (18) and (19) simultaneously. We shall show the existence of the solution of Eqs. (18) by finding them in the proof of St. 9. And the Eqns. (19), because of (17), are equivalent to (18).

St. 9. Let $\gamma_{0}:=\gamma_{1}+\gamma$ and $\gamma_{0}^{\prime}:=\gamma_{1}^{\prime}+\gamma^{\prime}, G\left(\gamma_{0}\right) Q$ and $G\left(\gamma_{0}^{\prime}\right) Q$ in Eq. (18) be rotations around the axes parallel to the unity vectors $d$, $d^{\prime}$, respectively. (The rotation angle in both cases is $\pi$.) The vectors $d, d^{\prime}$ are perpendicular to the $Z$-axis and

$$
\sin \gamma_{0}=-2 d_{1} d_{2}, \quad \cos \gamma_{0}=1-2 d_{1}^{2}=-1+2 d_{2}^{2} .
$$

$\left(d_{i}, i=1,2,3\right.$ - coordinates of $d$.)

Moreover the relation $H^{-1}\left(h^{\prime}\right) H(h) d=d^{\prime}$ follows from (18). Let us define $a_{i}:=\left(H^{-1}\left(h^{\prime}\right) H(h)\right)_{3 i}, i=1,2$. There occurs $d_{3}=d_{3}^{\prime}=0$, so $a_{1} d_{1}+a_{2} d_{2}=0$. Using this equality and the unity of vector $d$, we find $d_{1}$ and $d_{2}$ and then from Eq. (20) we obtain (15). The equality $a_{1}^{2}+a_{2}^{2}=0$ is equivalent to $h^{\prime}= \pm h$.

St.10. The line associated to $[h, y]$ is intersected by lines associated to $\left[h^{\prime}, y^{\prime}\right]$ and $\left[h^{\prime \prime}, y^{\prime \prime}\right]$ at the same point if and only if $\gamma_{0}^{\prime}=\gamma_{0}^{\prime \prime}$. This is equivalent to the equation

$$
a_{1}^{\prime} a_{2}^{\prime \prime}=a_{1}^{\prime \prime} a_{2}^{\prime}
$$

Let us assume first that $\theta \neq 0, \pi$, i.e. $h_{3}^{2} \neq 1$. In this case $a_{1}^{k}=\left(h^{k} \times h\right)_{3} t^{-1}, \quad a_{2}^{k}=\left(\left(h^{k} \times h\right) \times h\right)_{3} t^{-1}$, where $k==^{\prime}$, " and $t=$ $\left(1-h_{3}^{2}\right)^{1 / 2}$. Equation (21) expressed by the coordinates of the 
vectors $h, h^{\prime}, h^{\prime \prime}$ takes the form

$$
\left|\begin{array}{lll}
h_{1} & h_{2} & h_{3} \\
h_{1}^{\prime} & h_{2}^{\prime} & h_{3}^{\prime} \\
h_{1}^{\prime \prime} & h_{2}^{\prime \prime} & h_{3}^{\prime \prime}
\end{array}\right|\left(1-h_{3}^{2}\right)=0
$$

Then the determinant vanishes and vectors $h, h^{\prime}, h^{\prime \prime}$ are coplanar.

In the case when $h_{3}^{2}=1$ we have

$$
a_{1}^{k}=\sin \theta^{k} \sin \left(\psi-\psi^{k}\right),
$$

$a_{2}^{k}=\sin \theta^{k} \cos \left(\psi-\psi^{k}\right)$ for $\theta=0$ and $a_{2}^{k}=-\sin \theta^{k} \cos \left(\psi-\psi^{k}\right)$ for $\theta=\pi$. As the result of (16) there is

$$
\gamma_{0}^{k}=\pi+2\left(\psi-\psi^{k}\right)(\bmod 2 \pi), \text { when } \theta=0
$$

and

$$
\gamma_{0}^{k}=\pi-2\left(\psi-\psi^{k}\right)(\bmod 2 \pi), \text { when } \theta=\pi .
$$

Therefore, the condition $\gamma_{0}^{\prime}=\gamma_{0}^{\prime \prime}$ implies $\psi^{\prime}=\psi^{\prime \prime}$. Hence, $h, h^{\prime}, h^{\prime \prime}$ are coplanar in this case, too.

St.11 and 12. Similarly as in the proofs of statements 8 and 9 one gets the equations

$$
\begin{aligned}
H^{-1}\left(h^{\prime}\right) H(h) G\left(\gamma-\gamma_{2}\right)\left(H^{-1}\left(h^{\prime}\right) H(h)\right)^{-1} & =G\left(\gamma_{2}^{\prime}+\gamma^{\prime}\right) Q \\
Y\left(y^{\prime}\right) Y^{-1}(y) G\left(\gamma_{2}-\gamma\right)\left(Y\left(y^{\prime}\right) Y^{-1}(y)\right)^{-1} & =G\left(\gamma_{2}^{\prime}-\gamma^{\prime}\right) Q
\end{aligned}
$$

The occurrence of the intersection of lines $\left[h^{\prime}, y^{\prime}\right]$ and $\left[h^{\prime},-y^{\prime}\right]$ by the line $[h, y]$ is equivalent to the existence of the parameters $\gamma_{2}, \gamma_{2}^{\prime}$ satisfying (22) and (23). (22) occurs if and only if

$$
H^{-1}\left(h^{\prime}\right) H(h)\left[\begin{array}{c}
0 \\
0 \\
\pm 1
\end{array}\right]=\left[\begin{array}{c}
\cdot \\
\cdot \\
0
\end{array}\right] \text { i.e. } h \cdot h^{\prime}=0 .
$$

The angle of rotation $G\left(\gamma_{2}^{\prime}+\gamma^{\prime}\right) Q$ is $\pi$; so, the angle of $G\left(\gamma-\gamma_{2}\right)$ is also equal to $\pi$. Then $\gamma=\gamma_{2}+\pi(\bmod 2 \pi)$.

Equations (23) are equivalent to (22) if $\gamma=\gamma_{2}+k \pi$ and this holds in this case. 